\title{
Folate receptors and transporters: biological role and diagnostic/therapeutic targets in cancer and other diseases
}

\author{
Barbara Frigerio', Claudia Bizzoni ${ }^{1,7}$, Gerrit Jansen², Christopher P. Leamon ${ }^{3}$, Godefridus J. Peters ${ }^{4}$, Philip S. Low ${ }^{5}$, \\ Larry H. Matherly ${ }^{6+}$ and Mariangela Figini ${ }^{1 *+}$
}

\begin{abstract}
Folate receptors and transporters and one-carbon metabolism continue to be important areas of study given their essential roles in an assortment of diseases and as targets for treatment of cancer and inflammation. Reflecting this, every 2 years, the Folate Receptor Society organizes an international meeting, alternating between North America and Europe, where basic and translational scientists, clinical oncologists and rheumatologists from both academia and industry convene in an informal setting. The 7th International Symposium on Folate Receptors and Transporters was held in Sant'Alessio Siculo (ME), Taormina, Italy from 1st to 5th of October 2018, organized by Dr. Mariangela Figini from Fondazione IRCCS Istituto Nazionale dei Tumori, Milan. Following the format of previous meetings, more than 50 scientists from 9 different countries attended the 2018 meeting to share ongoing developments, discuss current research challenges and identify new avenues in basic and translational research. An important feature of this meeting was the participation of young investigators and trainees in this area, two (A. Dekhne and N. Verweij) of whom were awarded fellowships to attend this meeting as a recognition of the high scientific quality of their work. This report provides a synopsis of the highlights presented in the following sessions: Barton Kamen Lecture; Targeting one-carbon metabolism in cytosol and mitochondria; Structure and biology of the one-carbon solute transporters; Physiology and pathophysiology of folate receptors and transporters; Folate receptors for targeting tumors and inflammatory diseases; Conventional and new anti-folate drugs for treating inflammatory diseases and cancer; Imaging; Ongoing clinical trials; and Chimeric Antigen Receptor cell therapies of cancer.
\end{abstract}

Keywords: Folate, Folate receptors, Folate transporters, Cancer targeting, Inflammation targeting, One-carbon metabolism, Imaging, CAR T cell

\section{Barton Kamen lecture}

Professor Joseph Bertino (Cancer Institute of New Jersey, Rutgers University, USA) gave the lecture "The mitochondrial folate enzyme MTHFD2 as a target for anticancer therapy" in honor of the late Barton Kamen, a pioneering physician-scientist and major contributor to folate receptor (FR) biology for many decades. Dr. Kamen was a former trainee during his pediatric residency at Yale and a later colleague at the Cancer

\footnotetext{
* Correspondence: mariangela.figini@istitutotumori.mi.it

${ }^{\dagger}$ Larry H. Matherly and Mariangela Figini are contributed equally to this work.

'Dipartimento di Ricerca Applicata e Sviluppo Tecnologico, Fondazione

IRCCS Istituto Nazionale dei Tumori, Milan, Italy

Full list of author information is available at the end of the article
}

Institute of New Jersey and the Robert Wood Johnson Medical School, now part of Rutgers University. Dr. Bertino gave an overview of one-carbon (C1) metabolism, its compartmentalization in mitochondria and cytosol, and the promise of therapeutic targeting mitochondrial $\mathrm{C} 1$ metabolism in cancer. Mitochondrial C1 metabolism, provides glycine, $\mathrm{NAD}(\mathrm{P}) \mathrm{H}$, ATP and $\mathrm{C} 1$ units for cytosolic biosynthetic reactions [1]. Key enzymes in this pathway include serine hydroxymethyltransferase (SHMT) 2 and NAD-dependent methylene tetrahydrofolate dehydrogenase (MTHFD) 2, both of which are frequently upregulated in cancer [2]. Dr. Bertino noted that like certain other $\mathrm{C} 1$ enzymes, MTHFD2 has been reported in the nucleus where it co-localizes with DNA replication sites [3].

(c) The Author(s). 2019 Open Access This article is distributed under the terms of the Creative Commons Attribution 4.0 International License (http://creativecommons.org/licenses/by/4.0/), which permits unrestricted use, distribution, and 
The significance of this finding is still evolving. Moreover, adult and embryonic tissues express mitochondrial MTHFD2L, a bifunctional enzyme, homologous to MTHFD2, utilizing NAD/NADP as cofactor [4]. In his talk, he noted that MTHFD2 is highly expressed in rapidly replicating tumor cells but not in normal adult tissues, providing a strong rationale for targeting this enzyme for selective cancer treatment (see Fig. 1 right side). He noted that MTHFD2 is a bifunctional enzyme with both MTHFD and cyclohydrolase activities that distinguish it from the homologous trifunctional cytoplasmic enzyme MTHFD1 that includes dehydrogenase, cyclohydrolase and formyltetrahydrofolate synthetase activities. Bertino described approaches for the rational design of MTHFD2 inhibitors, drawing from structural studies of homologous enzymes including the human cytoplasmic MTHFD1 for which the bifunctional methylenetetrahydrofolate dehydrogenase/cyclohydrolase domain has been crystalized with an inhibitor (LY345899). Although no inhibitor for MTHFD2 has yet emerged, its overexpression in rapidly replicating tumor tissues but not in normal tissues and the finding that knockdown of MTHFD2 effects a strong antiproliferative response in tumor cells provide compelling rationale for targeting MTHFD2 in cancer $[5,6]$.

\section{Targeting one-carbon metabolism in cytosol and mitochondria}

Following Bertino's lecture, several speakers further discussed the subject of compartmentalization and targeting C1 metabolism. G. Ducker (University of Utah, USA) gave a presentation entitled "Isotope tracing of compartmentalized folate metabolism" in which he described analytical methods for characterizing the sub-cellular compartmentalization of $\mathrm{C} 1$ metabolism including the metabolic fluxes. The focus was on mass spectrometry-based isotope tracing techniques that have enabled determination of the precise localization of folate reactions within cells and have supported development of novel inhibitors of mitochondrial and cytosolic C1 reactions in cancer [7].

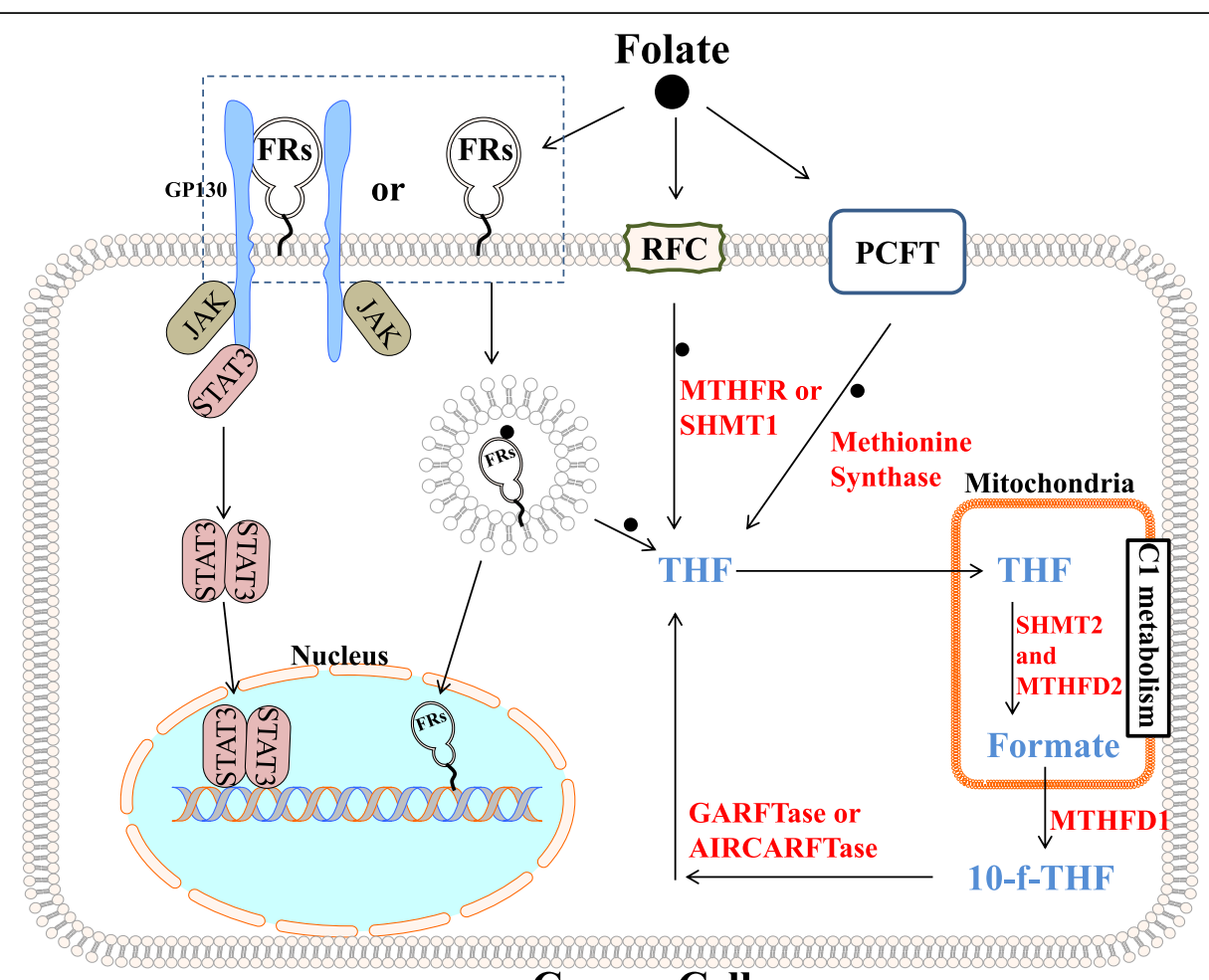

Cancer Cell

Fig. 1 Main aspects of folate receptor signaling and C1 metabolism discussed during the meeting. Three types of folate transporters/receptors are known to exist in humans to facilitate the uptake of folate: Folate Receptors (FRs), Reduced Folate Carrier (RFC) and Proton-Coupled Folate Transporter (PCFT) [57]. Left side: Folate binding to FRs can induce STAT3 activation via a GP130 co-receptor mediated JAK-dependent process. Folate can also bind FRs undergoing endocytosis and upon released FRs are set free to act like transcription factors [22]. Right side: Folate, through an interlinked set of mitochondrial and cytosolic reactions, support the C1 metabolism and the main pathway reactions are depicted $[1,6]$. THF: tetrahydrofolate; MTHFR, methylenetetrahydrofolate reductase; SHMT1/2, serine hydroxymethyl transferase in cytosol (1) and mitochondrial (2); MTHFD1, methylenetetrahydrofolate dehydrogenase 1; MTHFD2, methylenetetrahydrofolate dehydrogenase 2; 10-f-THF: 10-formyl- tetrahydrofolate; GARFTase: glycinamide ribonucleotide formyltransferase; AICARFTase: 5-aminoimidazole-4-carboxamide ribonucleotide formyltransferase 
A particular focus of several presentations was on the mitochondrial $\mathrm{C} 1$ metabolism enzyme, SHMT2, the first enzyme in serine catabolism that is induced upon hypoxic stress in Myc-transformed cells [8]. Expression of SHMT2 is highly correlated with the malignant phenotype across a broad spectrum of cancers, including lung, colon, breast, glioma, and liver [2]. Despite the unequivocal importance of SHMT2 to development and maintenance of the oncogenic phenotype, there are no clinically relevant inhibitors of this important target. A. Gangjee (Duquesne University, USA) presented the work "Design, synthesis and evaluation of first-in-class SHMT inhibitors" in which he described the design, molecular modeling, and synthesis of "first-in-class" 5-substituted pyrrolo [2,3- $d]$ pyrimidine compounds AGF291, AGF320, and AGF347 that inhibit the mitochondrial "oncodriver" SHMT2, along with cytosolic SHMT1, and C1 purine biosynthetic enzymes, glycinamide ribonucleotide formyltransferase (GARFTase) and/ or 5-aminoimidazole-4-carboxamide ribonucleotide formyltransferase (AICARFTase) [9]. L. Matherly (from the Barbara Ann Karmanos Cancer Institute, USA) presented "Discovery of novel pyrrolopyrimidine compounds that inhibit mitochondrial and cytosolic one-carbon metabolism with potent anti-tumor efficacy", demonstrating the potential of these novel compounds as broad-spectrum antitumor agents toward lung, colon and pancreatic tumors [10]. Antitumor efficacy reflected inhibition at mitochondrial $\mathrm{C} 1$ metabolism at SHMT2, and at cytosolic targets including $\mathrm{C} 1$-dependent purine biosynthesis and SHMT1, detected by targeted metabolomics with stable isotope tracers. Promising in vivo anti-tumor efficacy with curative potential was demonstrated in an aggressive pancreas cancer model. C. Dann III (Indiana University, USA) presented the "Biochemical and structural analysis of targeted cytotoxic therapeutics on folate utilizing enzymes in C1 metabolism" in which he described the enzymology and structural biology of target enzymes including GARFTase, AICARFTase/inosine monophosphate cyclohydrolase (ATIC), SHMT1, SHMT2 and MTHFD2 with the lead pyrrolo $[2,3-d]$ pyrimidine inhibitors [11]. His presentation described efforts to determine crystal structures of these enzyme inhibitor complexes to facilitate future drug design. Disparate activities of the lead molecules as cell-based inhibitors vis á vis isolated enzymes suggested an importance of cellular transport and polyglutamylation. Finally, A. Dekhne (Barbara Ann Karmanos Cancer Institute, USA) confirmed for the lead compound AGF347 (following radiolabeling) uptake by the reduced folate carrier (RFC), proton-coupled folate transporter (PCFT) and folate receptor (FR) $\alpha$, and mitochondrial accumulation and metabolism to AGF347 polyglutamates in both cytosol and mitochondria. Collectively, these studies establish the pyrrolo [2,3- $d$ ] pyrimidine analog AGF347 as an exciting prototype for multi-targeting mitochondrial and cytosolic $\mathrm{C} 1$ metabolism for cancer, with substantial promise for overcoming resistance to current anticancer therapies [12].

\section{Structure and biology of the one-carbon solute transporters}

Presentations by $\mathbf{M}$. Jansen (Texas Tech University Health Sciences Center, USA) and Z. Hou (Barbara Ann Karmanos Cancer Institute, USA) focused on the major folate transport system including the facilitative transporters PCFT and RFC, and FR $\alpha$. Both PCFT and FR $\alpha$ have been a focus for targeted drug delivery [10, 13], whereas RFC is the major tissue folate transporter for physiologic folates and clinically important antifolates such as methotrexate. The presentation by M. Jansen on the "Path to structural understanding of folate transport through PCFT" was aimed to understand PCFT function and determination of its atomic structure and molecular mechanism of transport. These approaches require large amounts of functional protein and several high-level heterologous expression systems were used for human PCFT including insect cells and yeast [14]. PCFT was functional in whole cell assays in both expression systems and after solubilization, purification to homogeneity, and reconstitution into liposomes. High-throughput crystallization trials identified conditions for PCFT crystallization that were systematically optimized to yield crystals suitable for high-resolution structure determination. The availability of a robust overexpression system for human PCFT provides a basis for future biochemical, biophysical and structural studies of this physiologically and pharmacologically important transporter.

Hou presented the "Impact of folate transport redundancies on cancer therapy with targeted and untargeted antifolates". The focus of his presentation was on functional inter-relationships (e.g., redundancies) among PCFT, FRs and RFC for novel tumor-targeted folate analogs (including those previously synthesized and studied by Gangjee and Matherly) such as AGF94 (a 6-substituted pyrrolo $[2,3-d]$ pyrimidine inhibitor transported by both PCFT and FRs but not RFC), AGF102 (FR-selective 6-substituted thienoyl [2,3- $d]$ pyrimidine analog, without substrate activity by PCFT or RFC), and classical antifolate drugs [pemetrexed (PMX), methotrexate $(\mathrm{MTX})]$ that are variably transported by $\mathrm{FR} \alpha$, PCFT and RFC [15]. Towards this goal, he described cell line models using a PCFT-, FR-, and RFC-null HeLa background engineered to express FR $\alpha$ or RFC under control of a tetracycline (Tet)-inducible promoter without or with constitutive expression of PCFT. Studies of transporter expression and function over a range of induction demonstrated that co-expression of the major 
folate transporters can result in transporter redundancies or antagonism with variable and surprisingly disparate impacts on anti-tumor efficacies of both classical and tumor-targeted antifolates depending on their transport specificities. The results identified critical determinants of anti-tumor activity with targeted and untargeted antifolates, including levels of transporter expression, intracellular folates, as well as extracellular $\mathrm{pH}$.

\section{Physiology and pathophysiology of folate receptors and transporters}

A number of presentations covered various aspects of the physiology and pathophysiology of FR and facilitative transporters. Professor Satyajit Mayor (National Centre for Biological Sciences, Bangalore, India) opened this session with the keynote lecture "Role of folate receptors in the organization of membranes of eukaryotic cells". Since 1994 Dr. Mayor focused his interest on FRs on the basis of the evidences provided by Kamen about intracellular FR trafficking [16]. Mayor has focused his work on FRs as prototypical glycosylphosphatidyl inositol (GPI)-anchored proteins with the goal of defining the role of the local structure and composition of the outer membrane in the control of membrane processes and mechanisms such as signaling, sorting, and exo- and endocytic processes. In his lecture, Mayor gave an overview of the physiology and pathophysiology of FRs and covered the mechanisms behind GPI-anchored proteins endocytosis and nanoclustering. Endocytic processing of GPI-anchored proteins, wherein they are preferentially endocytosed via a clathrin and dynamin-independent endocytic pathway, results in their uptake into an extremely acidic early endosomal compartment [17]. This is likely to be important for folate uptake. The unusual organization of the FR at the cell surface has sparked a new understanding of the specialized membrane microenvironment necessary for their function, where small nanoclusters of GPI-anchored proteins in a "sea" of monomers are generated in response to specific signals [18]. Detailed understanding of the physico-chemical principles behind the generation of these nanoclusters via the active mechanics of actin filaments and myosin is emerging. Studies of organization and trafficking of the GPI-anchored human FR provide unique insights into the endocytic pathways available at the cell surface to delineate a complex picture of membrane organization and how these machineries may be relevant for specific-FR functions and for their exploitation in imaging and targeted therapy $[19,20]$.

P. Martensen (Aarhus University, Denmark) gave a presentation "Folate receptor alpha and STAT3 activation" that extended her previous in vitro observations that folic acid can activate STAT3 through FR $\alpha$ in a Janus Kinase (JAK)-dependent manner, and that gp130 functions as a transducing receptor for this signaling in the FR $\alpha$-expressing tumor cells [21] (see Fig. 1, left side). Martensen also showed that breast tumors in female PyMT mice receiving a high folic acid diet displayed significantly increased tumour volumes along with STAT3 activation, compared to mice receiving a normal diet. Moreover, FR $\alpha$ knockdown abolished folic acid-induced STAT3 activation [22].

J. Kaur (Institute of Medical Education and Research, India) described in the presentation "Multiple Regulatory Mechanisms Control the Expression of Folate Transporters in Conditions of Ethanol Exposure and Folate Deficiency" her research on the regulation of the major folate transport systems in response to folate deficiency [23]. Folate deficiency can occur in both developing and well-developed countries with inadequate dietary folate intake and severe alcoholism as the most frequent causes. In a rat model of chronic ethanol feeding, folate transport decreased, accompanied by decreased expression of PCFT, RFC and FR in intestine, liver, colon and kidney, while increased mRNA expression of PCFT and RFC was recorded in pancreas. PCFT and RFC associated with membrane lipid rafts were decreased under conditions of chronic alcoholism. In response to dietary folate deficiency in rats, there was a significant decrease in folate levels, accompanied with increased uptake of folic acid across the membranes in intestinal and liver tissue and increased expression of folate transporters and FRs. A better understanding of the biological basis for physiologic folate deficiency in response to limiting dietary folate and chronic alcoholism will identify strategies for addressing this condition.

Folates are essential for brain development and function. Brain folate uptake primarily occurs at the choroid plexus through the concerted action of the FR $\alpha$ and PCFT. Inactivating mutations on FR $\alpha$ or PCFT cause cerebral folate deficiency, resulting in childhood neurodegeneration. The presentation by R. Bendayan (University of Toronto, Canada) "Up-regulation of reduced folate carrier by vitamin D enhances folate uptake at the blood-brain barrier" elaborated on the functional expression of RFC in brain microvessel endothelial cells representative of the blood-brain barrier (BBB) and its upregulation by the vitamin D nuclear receptor (VDR). The detection of RFC functional expression in various in vitro and in vivo BBB model systems suggests a potential role for this transporter in mediating brain folate permeability, especially when the predominant route of folate uptake at the choroid plexus is impaired. These data are the first to show that activation of VDR through calcitriol treatment can upregulate RFC functional expression at the BBB, implying that this transporter could potentially constitute a novel strategy for brain folate delivery and treating neurometabolic disorders caused by folate deficiency [24]. 
The regulation of folate transport in Cerebral Folate Deficiency (CFD) was the focus of the studies presented by R. Finnell and R. Cabrera (both from Center for Precision Environmental Health, USA) on "DNA binding transcriptional repression and the regulation of folate transport". CFD syndrome is defined as any neurological syndrome associated with low concentrations of 5-methyltetrahydrofolate in cerebrospinal fluid, while folate levels in plasma and red blood cells are within normal range. Previously, mutations in several folate pathway genes, including FR $\alpha$, dihydrofolate reductase, and PCFT were identified in CFD patients [25]. This now includes 70 individuals diagnosed with CFD who have been studied by genomic, whole exome sequencing and in vitro functional analyses. Clinical work-up of these patients failed to reveal any abnormalities in brain imaging but indicated disturbed cerebral folate transport. A de novo mutation in human Capicua gene (CIC), c.1057C > T (p.R353X), was identified in a patient that caused the protein to degrade through nonsense-mediated mRNA decay (NMD) such that less CIC protein is detected in the patient's fibroblast and iPS cells. A CIC target binding octamer sequence occurs in the promoter regions of the folate transport genes FR $\alpha$, PCFT, and RFC1, and dihydrofolate reductase, and binding was confirmed by chromatin immunoprecipitation assays. CIC conditional knockout mice showed similar phenotypes to CFD proband, including ASD-like behaviors and development delay. Thus, a fortuitous clinical interaction led to intensive mouse genetic studies that supported a causal interaction with CIC and CFD in a heretofore understudied regulatory mechanism of folate transport.

In cerebral folate deficiency folate transport across the choroid plexus is compromised. R. Steinfeld (University Children's Hospital Zurich, Switzerland) discussed in the presentation "Loss of folate receptor alpha function and cerebral folate deficiency" the role of FR $\alpha$ in folate uptake across the choroid plexus and the transport of folates from the cerebral spinal fluid (CSF) into the brain parenchyma [26]. At the basolateral (blood-site) of choroid plexus epithelial cells, FR $\alpha$ binds folates, after which the receptor is endocytosed into multivesicular bodies from which FR $\alpha$-containing vesicles/exosomes are formed and released in the CSF. From the CSF, FR $\alpha$-containing vesicles/exosomes are taken up by ependymal cells by a putative FR $\alpha$ binding protein (i.e. Low Density Lipoprotein Receptor-Related Protein 2 (megalin)) on ependymal cells to deliver folates to astrocytes and neurons. A better understanding of the cell biological pathways and proteins involved in cerebral folate transport will lead to identification of the defects that contribute to CFD.

\section{Folate receptors for targeting tumors and inflammatory diseases \\ Cancer}

Targeted therapies for cancer are aimed at maximizing tumor kill and minimizing toxicity. FRs were among the first validated targets for cancer since FRs are expressed in a subset of malignant cells and a limited number normal tissues, making FRs interesting Tumor Associated Antigens (TAAs) (see Fig. 2).

Monoclonal antibodies that target FR $\alpha$ on the surface of tumor cells are under evaluation in clinical trials [27-29]. They can mediate specific anti-tumor activity either by blocking cell signaling or by eliciting immune-mediated cell killing by engaging effector cells or complement. IgG antibodies are the commonly used class of antibodies in cancer therapy but other classes of antibodies are under evaluation. S. Karagiannis (King's College, UK) investigated in her presentation "Mechanisms of action for MOv18 IgE targeting folate receptor alpha-expressing tumours" whether antibodies engineered with Fc regions of IgE class may provide an alternative approach for treating solid tumours. This concept is based on the properties of this class to mediate immune clearance of parasites by effector cells such as macrophages. Unlike commonly-used IgG1 and IgG4, the IgE Fc regions feature very high affinities for cognate Fce-receptors on monocytes and macrophages, effector cells known to infiltrate tumours. Her group developed a first-in-class IgE antibody that recognizes FR $\alpha$ and exhibits superior tumour growth suppression compared with the corresponding IgG in two human tumour xenograft models. Efficacy was recapitulated in an immunocompetent syngeneic rat tumour model designed to more-closely mirror human IgE-FceR interactions. These results provide critical support for clinical translation of IgE as an anti-cancer strategy, and facilitated the launch of a clinical trial of this agent for patients with ovarian carcinoma tumours. This strategy may offer opportunities to extend the current IgG-only class of monoclonal antibodies for the treatment of solid tumours [27].

Targeting of pharmaceuticals is a rapidly evolving strategy to overcome the difficulties in therapeutic delivery, especially to the tumor site. Unlike traditional drug delivery systems, nanoparticle-based compounds attain superior uptake by tumors via active or passive mechanisms. Several presentations highlighted different preparative techniques and types of nanoparticles for tumor targeting through folate.

T. Pellegrino (Italian Institute of Technology, Italy) described in the presentation "Functionalized magnetic nanoparticles to target folate receptors to tackle cancer" her studies with magnetic nanoparticles that act as heat mediators under oscillating magnetic fields in the so-called "magnetic hyperthermia". The advent of 


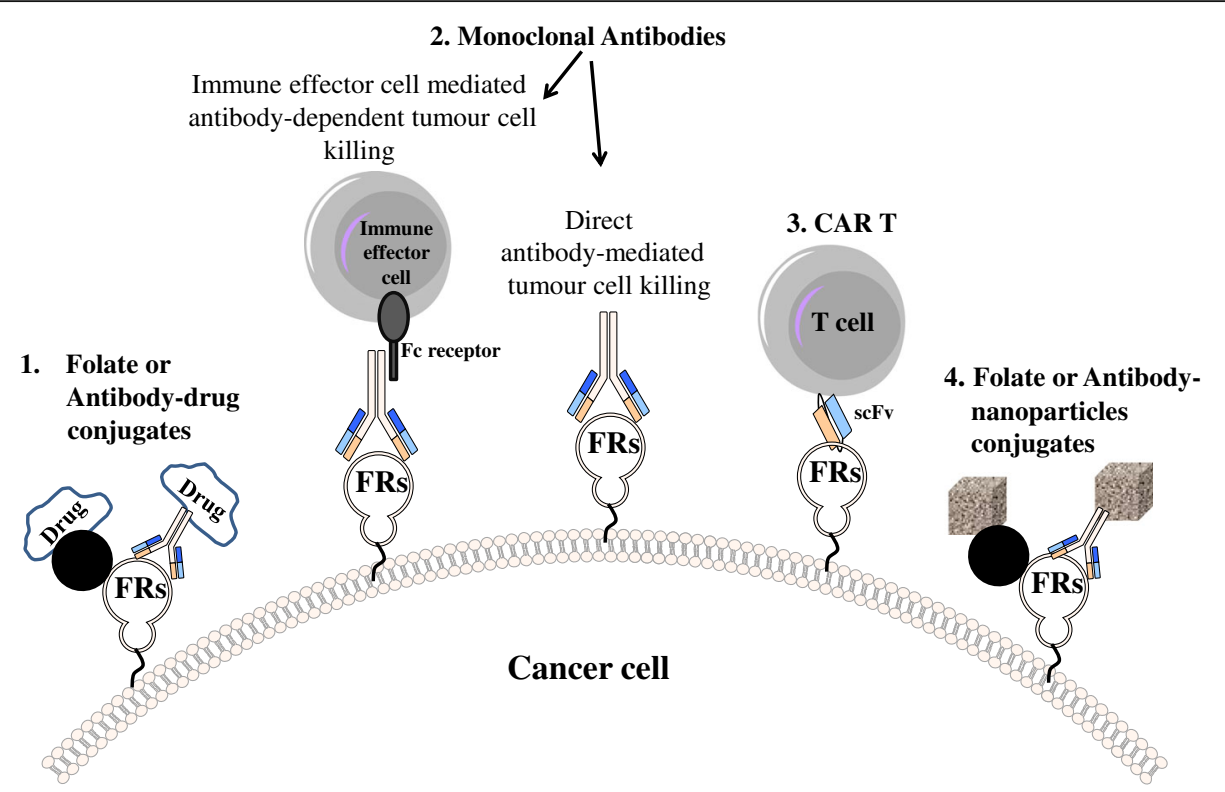

Fig. 2 Approaches for targeting FRs as therapies for cancer and inflammatory diseases. 1) Folate [13] or Antibody [58] drug-conjugates [59], 2) Monoclonal antibodies (immune effector cell engagement): antibodies link FRs-expressing tumor cells with immune effector cells that bear Fc receptors leading to antibody-dependent effector cell-mediated cytotoxicity [27, 29], 3) Chimeric antigen receptor (CAR) T cells: CAR T cells recognizing FRs through scFv (single chain variable fragment) trigger tumor cell killing [60-62], 4) Folate or Antibody nanoparticles-conjugates [63]

non-hydrolytic methods for preparing of magnetic nanoparticles has resulted in better control in terms of size, size distribution and crystallinity (parameters affecting structural and magnetic properties, thus their heat performance). Pellegrino focused on her recent progress in use of cubic-shaped iron oxide magnetic nanoparticles as a delivery tool for heat-mediated drug delivery (i.e., synthesis, functionalization, characterization, and drug loading) and in vitro and in vivo (both subcutaneous and orthotopic models) studies with targeting magnetic nanoparticles to FR $\alpha$-overexpressing ovarian cancer cells with anti-FR $\alpha$ antibody fragments [30]. Elemental and histological analyses showed that conjugated magnetic nanoparticles were specifically retained at tumor sites longer than the non-conjugated nanoparticles, suggesting their use as multifunctional theranostic agents [31].

S. Biocca (University of Roma "Tor Vergata", Italy) in "Selective targeting of folate-functionalized DNA nanocages" described DNA-based nanostructures for delivering classical chemotherapeutic agents [32, 33]. DNA possesses intrinsic properties of high stability, biocompatibility and versatility that makes it an extremely suitable polymer for generating nanoparticles. Dr. Biocca's group designed and assembled truncated DNA octahedral nanocages, functionalized with folate, to selectively target tumour cells overexpressing FR $\alpha$. Folate-DNA nanocages are internalized in FR+ tumour cells with efficiencies greater than 40 times compared to cells not expressing FRs and are taken up in a time-dependent manner with high intracellular stability $(>48 \mathrm{~h})$. While
DNA nanocages are not themselves cytotoxic, they can be loaded with doxorubicin (DOX) and following delivery to the cytoplasm and DOX release, result in a cytotoxic response.

Failure of chemotherapy can be due to Multidrug Resistance (MDR), by which cancer cells develop resistance towards different chemotherapeutic drugs, often with unrelated structures and distinct pharmacodynamic properties. A major mechanism of MDR involves overexpression of P-glycoprotein (Pgp) which actively extrudes drugs. As an approach to bypass drug efflux by Pgp, E. Gazzano (University of Torino, Italy) during the "Folate-targeted liposomal nitrooxy-doxorubicin: An effective tool against P-glycoprotein-positive and folate receptor-positive tumors" talk presented data about the synthesis and validation of the efficacy of an innovative liposomal formulation which encapsulates a DOX-derivative conjugated with a nitric oxide releasing group. Liposomes were functionalized with folic acid as tumor targeting "antenna", and their activity was evaluated in preclinical models of DOX-resistant breast tumors. This formulation revealed superior efficacy to DOX and Caelyx (commercial DOX liposomal formulation), and showed a cardiovascular safety profile similar to Caelyx ${ }^{\oplus}[34,35]$.

Folic acid conjugation to therapeutic molecules has been a bona fide approach for selective targeting of FR-positive cancer cells [36]. A poster presentation, "Synthesis of N4Py Derivatives Containing Targeting Groups for Tumor Cells", by R. de Vries (University of 
Groningen, The Netherlands) described synthesis of a folic acid conjugate of a mimic of the anti-tumor drug Bleomycin (BLM), N,N-bis (2-pyridylmethyl)-N-bis (2-pyridyl)-methylamine (N4Py) [37]. One of the conjugates (N4Py-S-S-FA) contained a disulfide bond, which can be reduced intracellularly to facilitate release of N4Py. In vitro experiments demonstrated that N4Py-S-S-FA elicited good selectivity toward FR $\alpha$-expressing $\mathrm{KB}$ and IGROV-1 tumor cells and is similarly cytotoxic as the parent iron complex of the ligand N4Py.

\section{Inflammation}

Immune cells involved in inflammatory processes (e.g., tumor- associated macrophages (TAMs) and chronic inflammatory/autoimmune diseases (e.g. rheumatoid arthritis (RA)) express the FR $\beta$ isoform. FR $\beta$ expression is restricted to cells of the myeloid lineage, i.e., monocytes and macrophages. RA patients are characterized by synovial infiltration of FR $\beta$-positive macrophages which can be imaged and/or targeted for therapy [38, 39]. Little is known about FR $\beta$ expression on macrophage precursors, i.e. circulating monocytes in peripheral blood. The presentation "FR $\beta$ expression profiles in RA blood and synovial tissue" by G. Jansen (VU University Medical Center, The Netherlands) showed work in progress indicating that in peripheral blood of RA patients, FR $\beta$ expression was highest on CD14 low/CD16 high subpopulation of monocytes.

\section{Conventional and new anti-folate drugs for treating inflammatory diseases and cancer}

Methotrexate (MTX) remains a first line of therapy for patients with rheumatoid arthritis (RA). However, despite its prominent use for more than 25 years, the mechanisms through which MTX suppresses inflammation in RA are not well understood. A. Puig Kröger (Instituto de Investigación Sanitaria Gregorio Marañón, Hospital General Universitario Gregorio Marañón, Spain) in "Unmasking the anti-inflammatory effects of methotrexate in arthritis" described the molecular impact of MTX on gene expression in inflammatory macrophages, a major cell type involved in the etiology of RA. In her presentation, Puig-Kröger demonstrated that low-dose MTX conditions macrophages towards a tolerant state by decreasing production of pro-inflammatory cytokines in response to pro-inflammatory stimulants (e.g. TNFo, LPS, RASF). MTX induces upregulation of the TNFAIP3 gene, which encodes for the protein A20, a negative regulator of $\mathrm{NFKB}$ signaling. By elevating expression of TNFAIP3, a gene already associated with reduced susceptibility to RA, the pro-inflammatory macrophage that is critical to maintenance of the inflammatory state in $\mathrm{RA}$ is reprogrammed to a quiescent or tolerant state.
Studies were described showing 1) that knockdown of A20 strongly reduces the tolerance-inducing effect of MTX, and 2) that TNFAIP3 expression in peripheral blood cells is significantly higher in RA patients who respond to treatment with MTX than those who don't. These studies establish a central role for upregulation of TNFAIP3 in the therapeutic mechanism of MTX in treatment of RA [40].

R. de Jonge and I.B. Muller (VU University Medical Center, The Netherlands) in their presentations "Methotrexate TDM in arthritis" described the design and optimization of a clinically applicable LC-MS/MS method for quantitation of methotrexate polyglutamate (MTX-PG) levels. They applied their method to monitor MTX-PG levels in three prospective cohort studies in adult and pediatric arthritis patients. Higher intracellular MTX-PGs were associated with improved clinical responses and inter-individual variations in MTX-PG levels were shown to be at least partly (20\%) explained by age, baseline erythrocyte-folate, a single nucleotide polymorphism in the gene for folylpolyglutamate synthetase, and MTX dose [41].

T. Ratliff's (Purdue University, USA) presentation "Targeting Myeloid Cells in the Tumor Microenvironment" described studies in mouse and human tumors to identify specific markers, including FR $\beta$, in tumor-associated fibroblast and myeloid-derived suppressor cells, which feature an immune-suppressive phenotype. Targeting of these cells can be an attractive strategy to induce antitumor activity [42].

Presentations by M.P. Costi (University of Modena and Reggio Emilia, Italy), and A.L. Jackman and U. Banerji (The Institute of Cancer Research, UK) described studies on cancers treated with novel anti-folate drugs. In "Dimer disrupters of thymidylate synthase (TS) through folate receptor targeting" Dr. Costi described several compounds that interfered with the dimeric assembly of the homodimeric enzyme, TS, a major therapeutic target for antifolates and fluoropyrimidines. Inhibition of TS dimerization results in inhibition of enzyme activity, suggesting that these represent a new class of chemotherapy agents that interfere with folate metabolism.

In "Preclinical and early clinical experience with BTG 945 (BGC945; ONX0801; CT900)" Jackman and Banerji presented exciting preclinical and clinical data on a new antifolate BTG945 (ONX0801). Recognizing that all FDA-approved antifolates enter cells via the RFC that is expressed in virtually all human cell types, and that FR $\alpha$ is expressed primarily on cancer cells with little-or-no expression on other human cell types except the proximal tubule cells of the kidneys, Jackman designed a new antifolate BTG945 that is transported into cells almost exclusively via FR $\alpha$. Once internalized by tumours 
over-expressing FR $\alpha$, BTG945 inhibits TS, resulting in anti-tumor activity. Preclinical pharmacokinetic and pharmacodynamic data defined the optimal blood drug levels for therapeutic efficacy in humans $\left(12 \mathrm{mg} / \mathrm{m}^{2}\right.$ every other week) and early clinical results demonstrated very promising activity in ovarian cancer patients with $\mathrm{FR} \alpha$ positive tumours ( $46 \%$ of the patients showed a GCIG CA125 response and 70\% displayed a Recist response). Ongoing trials are underway to establish the optimal dosing concentration and frequency with plans for a randomized Phase $2 / 3$ study in the near future [43].

\section{Imaging}

Oncogene-driven deregulation of energy metabolism in malignant cells was recognized in 2011 as an emerging hallmark of cancer [44]. F. Podo (in quiescence Istituto Superiore di Sanità, Italy) gave a presentation entitled "Cancer metabolic reprogramming: novel insights offered by molecular imaging" that described molecular imaging approaches for in vivo monitoring of altered metabolic pathways in cancer cells. These include: 1) activation of aerobic glycolysis by $\left[{ }^{18} \mathrm{~F}\right]$ fluorodeoxyglucose (FDG) positron emission tomography (PET) and hyperpolarized $\left[{ }^{13} \mathrm{C}\right]$ magnetic resonance spectroscopy (MRS)/magnetic resonance spectral imaging (MRSI); 2) deregulated phosphatidylcholine (PtdCho) cycle using $\left[{ }^{1} \mathrm{H}\right] \mathrm{MRS} / \mathrm{MRSI}$ and PET $\left[{ }^{11} \mathrm{C}\right]$ choline; and 3) the "flare" effect from folate-mediated TS inhibition on proliferation-dependent uptake of the PET imaging agent $\left[{ }^{18} \mathrm{~F}\right]$ FLT. Her presentation focused on molecular imaging approaches to monitor folate-dependent $\mathrm{C} 1$ metabolism in vivo and comparison of folate radiotracers for PET imaging using different radionuclides $\left({ }^{18} \mathrm{~F},{ }^{68} \mathrm{Ga},{ }^{44} \mathrm{Sc},{ }^{152} \mathrm{~Tb}\right)$. She concluded that $\left[{ }^{18} \mathrm{~F}\right]$ FLT PET is suitable for monitoring the effects of TS inhibitors in vivo, since it enables evaluation of the balance between the de novo and salvage pathways for generation of thymidylate $[45,46]$.

Presentations "Preclinical FR-macrophage PET guided therapy response monitoring" by C. Molthoff and "[18F]Fluoro-PEG-Folate PET Imaging in Rheumatoid arthritis patients" by N. Verweij (both from VU University Medical Center, The Netherlands) explored the utility of the PET tracer $\left[{ }^{18} \mathrm{~F}\right]$-fluoro-PEG-folate in quantifying the accumulation and retention of activated macrophages in the inflamed joints of patients with rheumatoid arthritis. Because cartilage degradation and bone erosion manifest in RA joints until successful treatment is initiated, early detection of RA is essential to prevent irreversible joint damage. Since activated macrophages constitute the major inflammatory cell type in RA lesions and these inflammatory macrophages (but not tissue resident macrophages in healthy tissues) express FR $\beta$ that can be imaged with $\left[{ }^{18} \mathrm{~F}\right]$ Fluoro-PEG-Folate, the authors hypothesized that the intensity of an
RA joint's inflammation might be assessed by quantitating its uptake of the folate-targeted PET tracer mentioned above. Molthoff provided data from a rat model of RA demonstrating that uptake of $\left[{ }^{18} \mathrm{~F}\right]$ Fluoro-PEGFolate was significantly reduced in the inflamed joints following MTX therapy than before. This diminished accumulation of imaging agent was due to decreased number of activated macrophages in these joints. The FR $\beta+$ macrophage numbers were also reduced in the liver and spleen of RA rats following MTX therapy, suggesting that MTX suppressed the numbers of activated macrophages both locally (in inflamed joints) and systemically. Verweij evaluated the ability of $\left[{ }^{18} \mathrm{~F}\right]$ Fluoro-PEG-Folate to image inflamed lesions in human patients with clinically diagnosed RA. Data from a phase 1 clinical trial demonstrated that the PET tracer specifically concentrated in the inflamed lesions of patients with RA, yielded better images of inflamed lesions in whole body scans better than the reference macrophage tracer because of a lower background signal and is safe for use in humans [47].

Another important use of folate for targeted imaging of FR-positive lesions was presented by $\mathbf{P}$. Low (Purdue University, USA) in "Tumor-targeted NIR dyes for fluorescence-guided surgery". With the goal of improving the ability to locate and completely remove malignant lesions during surgery, his research group developed tumor-targeted folate-targeted fluorescent dyes, a folate-fluorescein (EC17) and a folate-targeted near infrared dye (OTL38), that selectively bind to FRs on cancer cells and can be detected upon excitation with the proper light source. Within $2 \mathrm{~h}$ of intravenous injection, the targeted fluorescent dyes clear from healthy tissues and accumulate in malignant tissues, allowing facile distinction of tumor from adjacent healthy tissues. Results were presented for patients with lung, brain, kidney, ovarian and other cancers. Results presented by Low demonstrated that the targeted fluorescent dyes were highly specific for tumor tissue and that use of these dyes during surgery enabled surgeons to 1) find and remove more malignant lesions, positive tumor margins, malignant lymph nodes much more efficiently than was previously possible using standard surgical methods (i.e., palpation and visual inspection); 2) more accurately stage cancer patients. With the aid of new and improved NIR cameras currently under development, these tumor-targeted NIR dyes should significantly expand the tools available for fluorescence-guided surgery and increase the chances of complete tumor resection and consequent cancer patient survival [48].

\section{Ongoing clinical trials}

This session began with Y. Setiady (ImmunoGen, USA) presenting the "Development of IMGN853, a folate 
receptor $\alpha$ (FR $\alpha)$ targeting antibody-drug conjugate $(A D C)$, for ovarian cancer treatment". IMGN853 (mirvetuximab soravtansine) is an antibody-drug conjugate (ADC) comprised of the chimeric anti-FR $\alpha$ M9346A antibody, a humanized derivative of the previously described anti-FRa antibody Mov19 [49] linked to the tubulin-disrupting maytansinoid, DM4, via a sulfo-SPDB linker. IMGN853 binds to FR $\alpha$ on cancer cells and is subsequently internalized [50]; DM4 is released from the antibody through both enzymatic degradation and/or disulfide linker cleavage, resulting in disruption of cell division, followed by cell death. Phase 1 clinical data showed that IMGN853 has promising single-agent activity and a favorable safety profile against FR $\alpha$-positive platinum-resistant ovarian cancer patients. IMGN853 is currently being evaluated in FORWARD I, a Phase 3 monotherapy study, as well as in combination with other agents (including pembrolizumab; anti-PD1 antibody) in a Phase $1 \mathrm{~b} / 2$ study, called FORWARD II.

C. Leamon (Endocyte Inc., USA) followed with a presentation entitled "Translational struggles of folate SMDCs: New learning and future directions" in which he provided a summary spanning nearly 20 years of cancer-targeted small molecule drug conjugate research, with special emphasis on EC1456, a folate-tubulysin conjugate that was tested in a phase 1 trial [51]. Leamon reviewed a comprehensive dataset involving ovarian cancer lesion analysis, correlating immunohistochemical (IHC) staining results to the uptake of ${ }^{99 \mathrm{~m}} \mathrm{Tc}$-etarfolatide (a SPECT-based imaging probe for functional FR expression) [52]. He also showed unpublished data on the quantization of EC1456 uptake, as well as associated metabolites in those same lesions.

As the role of biomarkers in drug discovery, development and clinical trials has gained increased importance there is great emphasis on quality assurance and assay validation so as to establish standardized guidelines for analytical methods used in biomarker measurements. The establishment of a concrete validation process that addresses technology, integration and method validation, as well as regulatory pathways for efficient biomarker development, is fundamental. In this context, E. Somers (Morphotek Inc., USA), Y. Wettergren (Institute of Clinical Sciences, Sweden) and G. Peters (VU University Medical Center, The Netherlands) described approaches for selecting patients for therapeutic trials, including the use of folate transporters such as FR $\alpha$ or PCFT as potential biomarkers. For instance, Somers described in "Development and validation of the folate receptor IHC assay" an IHC assay for selecting patients for inclusion in Morphotek's therapeutic trials involving FR $\alpha$ targeting. Their monoclonal antibody was licensed non-exclusively to Biocare Medical (Concord, CA) to enable manufacturing and distribution of IHC kits for research applications.
The kit was developed in a manual use format, and for use on Biocare IntelliPath ${ }^{\mathrm{rat}}$, Dako Link and Leica Bond instruments. Studies evaluated the frequency and expression levels of FR $\alpha$ in multiple tumor types to enable decisions regarding tumor targets and histologic subtypes. Kits were developed in two formats, a multi-tumor kit and an ovarian tumor kit, to extend their use to multiple tumor types with a broad range of FR expression. Based on discussion with the US FDA, this assay is currently being validated for use in selecting patients for planned clinical trials.

Wettergren in "The use of folate transporters as biomarkers in colorectal cancer therapy" reported high expression of SLC46A1/PCFT, SLC19A1/RFC-1, and $\mathrm{ABCC} / \mathrm{MRP} 3$ was associated with longer 5-year disease-free survival (DFS) of patients with colorectal cancer $(n=363)$ treated with adjuvant 5 -fluorouracil plus leucovorin (FLV), whereas there was no association between expression and DFS in untreated patients [53]. Furthermore, a positive association between 3-year progression-free survival and ABCC3/MRP3 expression was found in patients with advanced colorectal cancer $(n=294)$. She concluded that the folate transporter genes may have predictive value for patients with colorectal cancer going through FLV-based treatment.

Peters presented the "Role of PCFT in PMX resistance of malignant mesothelioma: update of clinical evidence and new pharmacological tools". Here, he identified PCFT as a novel biomarker in malignant mesothelioma. In two cohorts totaling 124 patients treated with PMX and carboplatin, high expression (either protein or mRNA) of PCFT was associated with a longer overall survival (OS) of patients, while low TS expression was also associated with a longer survival. Furthermore, a combination of low PCFT/high TS resulted in very poor survival. Notably, RFC expression was not related to OS. He concluded that PCFT and TS can be considered predictive biomarkers for malignant mesothelioma and should be tested prospectively to select patients who would be most responsive to PMX-based therapies [54].

\section{Chimeric antigen receptor cell therapies of cancer}

Chimeric Antigen Receptor (CAR T) cell-based therapies have transformed pediatric oncology by producing high remission rates and durable responses in CD19+ B-cell malignancies. This scenario is ideal as CD19 expression is homogeneous and human blood provides a favorable environment for CAR T cells to thrive and destroy cancer cells (along with normal B cells). However, CAR T cell therapies for solid tumors remain a challenge due to problems with tumor heterogeneity, cytokine release syndrome (CRS) and CAR $\mathrm{T}$ cell exhaustion. Several talks reported exciting new approaches in this promising field. 
D. Powell (University of Pennsylvania, USA) already presented in the previous edition of this meeting a FR $\alpha$ CAR T cells able to efficiently eliminate aggressive ovarian cancer xenografts in a murine subcutaneous tumor model [55]. Based on very promising safety and efficacy data, plans for a human clinical trial are underway (NCT03585764). This year his presentation "CAR T anti $F R$ beta" described the development of a CAR T cell products targeting FR $\beta$ for the elimination of acute myeloid leukemia (AML) blasts and tumor-associated macrophages (TAMs) in the tumor microenvironment. In a review of earlier published results, he showed that FR $\beta$-specific CAR T cells eradicated AML xenografts in mice [56] and summarized studies in which the same FR $\beta$ CAR T cells selectively eliminated immunosuppressive FR $\beta$ + TAMs, resulting in control of cancer progression in a syngeneic mouse model of an FR $\beta$-negative ovarian cancer. Based on these results, a combination therapy that directly eliminates cancer cells together with a CAR T cell therapy that can eradicate the FR $\beta+$ TAMs should be highly synergistic.

P. Low (Purdue University, USA) in "A universal CAR $T$ cell technology that avoids most limitations of current therapies" introduced a strategy for a universal CAR T therapy designed to avoid limitations known to compromise current CAR T cell therapies. These include: 1) toxicities arising from excessive release of cytokines (CRS); 2) resistance deriving from loss of the targeted tumor antigen; 3) exhaustion stemming from chronic exposure to tumor antigen; and 4) the need to engineer a different CAR $\mathrm{T}$ cell for each antigenically distinct tumor. Low described a CAR T cell technology that required only a single important modification. Briefly, he has engineered a CAR that contains the usual activation motifs (CD3 $\zeta$ and 4-1BB), but expresses a scFv against fluorescein instead of the usual $\mathrm{scFv}$ against a tumor antigen. The resulting CAR T cell kills cancer cells and proliferates only when it encounters a cancer cell that labeled with fluorescein isothiocyanate (FITC). For the latter, they constructed a FITC-folate conjugate (bispecific adapter) designed to bridge between the anti-FITC CAR T cell and the FR-expressing cancer cell. Upon establishment of this bridge, the CAR T cell both proliferates and kills the cancer cell. He demonstrated that a variety of solid tumors in NOD SCID gamma mice could be eradicated upon addition of FITC-folate. Moreover, when symptoms of a CRS became severe, these could be suppressed either temporarily by interrupting adaptor administration, or by injecting excess fluorescein to block adaptor bridging. To prevent disease recurrence that can arise if a cancer cell mutates to delete the targeted antigen, he administered a cocktail of low molecular weight bispecific adapters, each comprised of fluorescein linked to a different tumor-specific ligand that recognized one of the remaining tumor antigens. Finally, to avoid CAR $\mathrm{T}$ cell exhaustion associated with chronic exposure of CAR T cells to antigen, he periodically deleted bispecific adapter addition.

J. Lu (Endocyte Inc., USA) in "Preclinical Evaluation of Bispecific Adaptor Molecule Controlled Folate Receptor CAR T Cell Therapy" presented a more detailed examination of the CAR T adaptor molecule platform. She found that maximal CAR $\mathrm{T}$ cell cytolytic activity correlated with: 1) the level of expression of FR on the cancer cell's surface; 2 ) the number of CAR T cells that accumulated within the solid tumor; 3 ) the concentration of bispecific adapter (EC17, folate-fluorescein); 4) prevention of CAR T cell exhaustion; and 5) unidentified immunosuppressive factors within the tumor microenvironment. It was concluded that with proper optimization, this novel strategy could address several unmet needs in the CAR T therapy field.

\section{Closing remarks}

Altogether, the research presented at 7th meeting of the Folate Receptor Society highlighted the continued relevance of folate receptors/transporters in cancer and other diseases and the up-coming clinical exploitations of specific drugs and biological tools. In addition, the success of the 2018 meeting strengthens the Folate Receptor Society in promoting its important mission. These include: to foster sharing and discussion of the ongoing developments in the areas of folate receptors/ transporters and one-carbon metabolism; and to organize every 2 years an international meeting in which distinguished and trainee scientists with different expertise can meet in an informal way to discuss current research challenges and to identify new avenues in basic and translational research. We thank the organizers, sponsors, and all the participants who made the 2018 meeting a tremendous success and we look forward to the 8th meeting of the Folate Receptor Society which will be held in Austin, Texas in 2020.

\footnotetext{
Abbreviations

$\left[{ }^{18} \mathrm{~F}\right] \mathrm{FLT}$ : Fluorothymidine $\mathrm{F}^{-18}$; ADC: Antibody drug conjugate; AICARFTase: 5-aminoimidazole-4-carboxamide ribonucleotide formyltransferase; AML: Acute myeloid leukemia; ATIC: inosine monophosphate cyclohydrolase; BBB: Blood-brain barrier; BLM: Bleomycin; C1: one-carbon; CAM: CAR T adaptor molecule; CAR: Chimeric Antigen Receptor; CFD: Cerebral Folate Deficiency; CIC: human Capicua gene; CRS: Cytokine release syndromes; CSF: Cerebral spinal fluid; DFS: Disease-free survival; DOX: Doxorubicin; FITC: Fluorescein isothiocyanate; FR: Folate receptor; GARFTase: Glycinamide ribonucleotide formyltransferase; GPI: Glycosylphosphatidyl inositol; IHC: Immunohistochemical; JAK: Janus Kinase; LRP2: Low Density Lipoprotein Receptor Related Protein 2; mAbs: Monoclonal antibodies; MDR: Multidrug Resistance; MTHFD: Methylene tetrahydrofolate dehydrogenase; MTHFD2L: homologous to MTHFD2; MTHFR: Methylenetetrahydrofolate reductase; MTX: Methotrexate; MTX-PG: Methotrexate polyglutamates; N4Py: N,N-bis (2pyridylmethyl)-N-bis (2-pyridyl)-methylamine; NIR: Near-infrared; NMD: Nonsense-mediated mRNA decay; OS: Overall survival; PCFT: Protoncoupled folate transporter; Pgp: P-glycoprotein; PMX: Pemetrexed;
} 
PtdCho: Phosphatidylcholine; RA: Rheumatoid arthritis; RFC: Reduced folate carrier; SHMT: Serine hydroxymethyltransferase; TAA: Tumor Associated Antigen; TAMs: Tumor associated macrophages; TCT: targeted chemotherapy or alternatively targeted conventional therapy; THF: Tetrahydrofolate;

TS: Thymidylate synthase; VDR: Vitamin D nuclear receptor

\section{Acknowledgements}

We thank Silvana Canevari (Fondazione IRCCS Istituto Nazionale dei Tumori, Italy) for critical reading of the manuscript and Nadia Daniel (Barbara Ann Karmanos Cancer Institute, USA) for help in the meeting organization.

\section{Funding}

This meeting was partially funded by Barbara Ann Karmanos Cancer Institute, Endocyte Inc., Fondazione IRCCS Istituto Nazionale dei Tumori, Immunogen, Isofol, Merck, Purdue University, and Folate Receptor Society.

\section{Availability of data and materials}

Not applicable.

\section{Author's contributions}

All authors contributed in the revision of the manuscript on the basis of their field of interest and approved the final version.

\section{Ethics approval and consent to participate}

Not applicable.

\section{Consent for publication}

Not applicable.

\section{Competing interests}

CPL is an employee of Endocyte, a Novartis company. PSL is a founder, stockholder and Chief Scientific Officer of On Target Laboratories LLC.

\section{Publisher's Note}

Springer Nature remains neutral with regard to jurisdictional claims in published maps and institutional affiliations.

\section{Author details}

'Dipartimento di Ricerca Applicata e Sviluppo Tecnologico, Fondazione IRCCS Istituto Nazionale dei Tumori, Milan, Italy. ${ }^{2}$ Amsterdam Rheumatology and Immunology Center, Amsterdam University Medical Center, location Vrije Universiteit, Amsterdam, The Netherlands. ${ }^{3}$ Endocyte Inc, West Lafayette, Indiana, USA. ${ }^{4}$ Department of Medical Oncology, VU University Medical Center, Cancer Center Amsterdam, Amsterdam, The Netherlands. ${ }^{5}$ Purdue University Institute for Drug Discovery, West Lafayette, Indiana, USA. ${ }^{6}$ Barbara Ann Karmanos Cancer Institute and Wayne State University School of Medicine, Detroit, MI, USA. ${ }^{7}$ Present address: ASST Papa Giovanni XXIII, Bergamo, Italy.

\section{Received: 28 January 2019 Accepted: 28 February 2019}

Published online: 12 March 2019

\section{References}

1. Ducker GS, Rabinowitz JD. One-carbon metabolism in health and disease. Cell Metab. 2017;25:27-42.

2. Nilsson R, Jain M, Madhusudhan N, Sheppard NG, Strittmatter L, Kampf C, et al. Metabolic enzyme expression highlights a key role for MTHFD2 and the mitochondrial folate pathway in cancer. Nat Commun. 2014;5:3128.

3. Gustafsson Sheppard N, Jarl L, Mahadessian D, Strittmatter L, Schmidt A, Madhusudan N, et al. The folate-coupled enzyme MTHFD2 is a nuclear protein and promotes cell proliferation. Sci Rep. 2015;5:15029.

4. Shin M, Bryant JD, Momb J, Appling DR. Mitochondrial MTHFD2L is a dual redox cofactor-specific methylenetetrahydrofolate dehydrogenase/ methenyltetrahydrofolate cyclohydrolase expressed in both adult and embryonic tissues. J Biol Chem. 2014;289:15507-17.

5. Tedeschi PM, Scotto KW, Kerrigan J, Bertino JR. MTHFD2--a new twist? Oncotarget. 2016;7:7368-9.

6. Tedeschi PM, Vazquez A, Kerrigan JE, Bertino JR. Mitochondrial methylenetetrahydrofolate dehydrogenase (MTHFD2) overexpression is associated with tumor cell proliferation and is a novel target for drug development. Mol Cancer Res. 2015;13:1361-6.
7. Ducker GS, Chen L, Morscher RJ, Ghergurovich JM, Esposito M, Teng X, et al. Reversal of cytosolic one-carbon flux compensates for loss of the mitochondrial folate pathway. Cell Metab. 2016;23:1140-53.

8. Ye J, Fan J, Venneti S, Wan YW, Pawel BR, Zhang J, et al. Serine catabolism regulates mitochondrial redox control during hypoxia. Cancer Discov. 2014:4:1406-17.

9. Ravindra M, Wallace-Povirk A, Karim MA, Wilson MR, O'Connor C, White K, et al. Tumor targeting with novel Pyridyl 6-substituted Pyrrolo [2,3-d] pyrimidine Antifolates via cellular uptake by folate receptor $a$ and the proton-coupled folate transporter and inhibition of De novo purine nucleotide biosynthesis. J Med Chem. 2018;61:2027-40.

10. Matherly LH, Hou Z, Gangjee A. The promise and challenges of exploiting the proton-coupled folate transporter for selective therapeutic targeting of cancer. Cancer Chemother Pharmacol. 2018;81:1-15.

11. Deis SM, Doshi A, Hou Z, Matherly LH, Gangjee A, Dann CE. 3rd. Structural and enzymatic analysis of tumor-targeted Antifolates that inhibit glycinamide ribonucleotide Formyltransferase. Biochemistry. 2016;55:4574-82.

12. Nayeen MJ, Shah K, Gangjee A, Dekhne A, Hou Z, Matherly LH. Abstract 1656: first-in-class, fluorinated folate receptor specific agents that target tumor cells via inhibition of serine hydroxymethyltransferase 2 (SHMT2) and 5-aminoimidazole4-carboxamide formyltransferase (AICARFTase). Cancer Res. 2018;78:1656.

13. Assaraf $Y G$, Leamon $C P$, Reddy JA. The folate receptor as a rational therapeutic target for personalized cancer treatment. Drug Resist Updat. 2014;17:89-95.

14. Date SS, Fiori MC, Altenberg GA, Jansen M. Expression in Sf9 insect cells, purification and functional reconstitution of the human proton-coupled folate transporter (PCFT, SLC46A1). PLoS One. 2017:12:e0177572.

15. Hou Z, Gattoc L, O'Connor C, Yang S, Wallace-Povirk A, George C, et al. Dual targeting of epithelial ovarian Cancer via folate receptor a and the protoncoupled folate transporter with 6-substituted Pyrrolo [2,3-d] pyrimidine Antifolates. Mol Cancer Ther. 2017;16:819-30.

16. Mayor S, Rothberg KG, Maxfield FR. Sequestration of GPI-anchored proteins in caveolae triggered by cross-linking. Science. 1994;264:1948-51.

17. Mayor S, Parton RG, Donaldson JG. Clathrin-independent pathways of endocytosis. Cold Spring Harb Perspect Biol. 2014;6. https://doi.org/10.1101/ cshperspect.a016758.

18. Varma R, Mayor S. GPl-anchored proteins are organized in submicron domains at the cell surface. Nature. 1998;394:798-801.

19. Gowrishankar K, Ghosh S, Saha S, C R, Mayor S, Rao M. Active remodeling of cortical actin regulates spatiotemporal organization of cell surface molecules. Cell. 2012;149:1353-67.

20. Raghupathy R, Anilkumar AA, Polley A, Singh PP, Yadav M, Johnson C, et al. Transbilayer lipid interactions mediate nanoclustering of lipid-anchored proteins. Cell. 2015;161:581-94.

21. Hansen MF, Greibe E, Skovbjerg S, Rohde S, Kristensen ACM, Jensen TR, et al. Folic acid mediates activation of the pro-oncogene STAT3 via the folate receptor alpha. Cell Signal. 2015;27:1356-68.

22. Hansen MF, Jensen SØ, Füchtbauer E, Martensen PM. High folic acid diet enhances tumour growth in PyMT-induced breast cancer. Br J Cancer. 2017;116:752-61.

23. Thakur S, More D, Rahat B, Khanduja KL, Kaur J. Increased synthesis of folate transporters regulates folate transport in conditions of ethanol exposure and folate deficiency. Mol Cell Biochem. 2016;411:151-60.

24. Alam C, Hoque MT, Finnell RH, Goldman ID, Bendayan R. Regulation of reduced folate carrier (RFC) by vitamin $D$ receptor at the blood-brain barrier. Mol Pharm. 2017;14:3848-58.

25. Watkins D, Rosenblatt DS. Update and new concepts in vitamin responsive disorders of folate transport and metabolism. J Inherit Metab Dis. 2012;35:665-70

26. Grapp M, Wrede A, Schweizer M, Huwel S, Galla HJ, Snaidero N, et al. Choroid plexus transcytosis and exosome shuttling deliver folate into brain parenchyma. Nat Commun. 2013:4:2123.

27. Karagiannis SN, Josephs DH, Bax HJ, Spicer JF. Therapeutic IgE antibodies: harnessing a macrophage-mediated immune surveillance mechanism against Cancer. Cancer Res. 2017;77:2779-83.

28. Ponte JF, Ab O, Lanieri L, Lee J, Coccia J, Bartle LM, et al. Mirvetuximab Soravtansine (IMGN853), a folate receptor alpha-targeting antibody-drug conjugate, potentiates the activity of standard of care therapeutics in ovarian Cancer models. Neoplasia. 2016;18:775-84.

29. Ledermann JA, Canevari S, Thigpen T. Targeting the folate receptor: diagnostic and therapeutic approaches to personalize cancer treatments. Ann Oncol. 2015;26:2034-43. 
30. Di Corato R, Bigall NC, Ragusa A, Dorfs D, Genovese A, Marotta R, et al. Multifunctional nanobeads based on quantum dots and magnetic nanoparticles: synthesis and cancer cell targeting and sorting ACS Nano. 2011:5:1109-21.

31. Quarta A, Bernareggi D, Benigni F, Luison E, Nano G, Nitti S, et al. Targeting FR-expressing cells in ovarian cancer with fab-functionalized nanoparticles: a full study to provide the proof of principle from in vitro to in vivo. Nanoscale. 2015;7:2336-51.

32. Raniolo S, Vindigni G, Unida V, Ottaviani A, Romano E, Desideri A, et al. Entry, fate and degradation of DNA nanocages in mammalian cells: a matter of receptors. Nanoscale. 2018;10:12078-86.

33. Raniolo S, Vindigni G, Ottaviani A, Unida V, lacovelli F, Manetto A, et al. Selective targeting and degradation of doxorubicin-loaded folatefunctionalized DNA nanocages. Nanomedicine. 2018;14:1181-90.

34. Riganti C, Rolando B, Kopecka J, Campia I, Chegaev K, Lazzarato L, et al. Mitochondrial-targeting nitrooxy-doxorubicin: a new approach to overcome drug resistance. Mol Pharm. 2013;10:161-74.

35. Pedrini I, Gazzano E, Chegaev K, Rolando B, Marengo A, Kopecka J, et al. Liposomal nitrooxy-doxorubicin: one step over caelyx in drug-resistant human cancer cells. Mol Pharm. 2014;11:3068-79.

36. Srinivasarao M, Galliford CV, Low PS. Principles in the design of ligandtargeted cancer therapeutics and imaging agents. Nat Rev Drug Discov. 2015;14:203-19.

37. Li Q, van der Wijst MG, Kazemier HG, Rots MG, Roelfes G. Efficient nuclear DNA cleavage in human cancer cells by synthetic bleomycin mimics. ACS Chem Biol. 2014;9:1044-51.

38. van der Heijden JW, Oerlemans R, Dijkmans B, Qi H, Laken C, Lems W, et al. Folate receptor beta as a potential delivery route for novel folate antagonists to macrophages in the synovial tissue of rheumatoid arthritis patients. Arthritis Rheum. 2009;60:12-21.

39. Chandrupatla DMSH, Molthoff CFM, Lammertsma AA, van der Laken, Jansen G. The folate receptor $\beta$ as a macrophage-mediated imaging and therapeutic target in rheumatoid arthritis. Drug Deliv Transl Res. 2019;9:366-78.

40. Municio C, Dominguez-Soto A, Fuentelsaz-Romero S, Lamana A, Montes N, Cuevas VD, et al. Methotrexate limits inflammation through an A20dependent cross-tolerance mechanism. Ann Rheum Dis. 2018;77:752-9.

41. de Rotte MCFJ, Pluijm SMF, de Jong PHP, Bulatović Ćalasan M, Wulffraat NM, Weel AEAM, et al. Development and validation of a prognostic multivariable model to predict insufficient clinical response to methotrexate in rheumatoid arthritis. PLoS One. 2018;13:e0208534.

42. Haverkamp JM, Crist SA, Elzey BD, Cimen C, Ratliff TL. In vivo suppressive function of myeloid-derived suppressor cells is limited to the inflammatory site. Eur J Immunol. 2011;41:749-59.

43. Banerji U, Ingles Garces AH, Michalarea V, Ruddle R, Raynaud Fl, Riisnaes R, et al. An investigator-initiated phase I study of ONX-0801, a first-in-class alpha folate receptor targeted, small molecule thymidylate synthase inhibitor in solid tumors. J Clin Oncol. 2017:35:2503.

44. Hanahan D, Weinberg RA. Hallmarks of cancer: the next generation. Cell. 2011:144:646-74.

45. Soloviev D, Lewis D, Honess D, Aboagye E. [(18) F]FLT: an imaging biomarker of tumour proliferation for assessment of tumour response to treatment. Eur J Cancer. 2012;48:416-24.

46. Frings $V$, van der Veldt AAM, Boellaard R, Herder GJM, Giovannetti E, Honeywell R, et al. Pemetrexed induced thymidylate synthase inhibition in non-small cell lung cancer patients: a pilot study with 3'-deoxy-3'-[18F] fluorothymidine positron emission tomography. PLoS One. 2013;8:e63705.

47. Verweij N, Bruijnen S, Gent Y, Huisman M, Jansen G, Molthoff C, Chen Q, Low P, Windhorst A, Lammertsma A, Hoekstra O, Voskuyl A, van der Laken C. Rheumatoid Arthritis Imaging on PET-CT Using a Novel Folate Receptor Ligand for Macrophage Targeting [abstract]. Arthritis Rheumatol. 2017; 69(suppl 10). https://acrabstracts.org/abstract/rheumatoid-arthritis-imagingon-pet-ct-using-a-novel-folate-receptor-ligand-for-macrophage-targeting/.

48. Mahalingam SM, Kularatne SA, Myers CH, Gagare P, Norshi M, Liu X, et al. Evaluation of novel tumor-targeted near-infrared probe for fluorescenceguided surgery of Cancer. J Med Chem. 2018;61:9637-46

49. Figini M, Obici L, Mezzanzanica D, Griffiths A, Colnaghi MI, Winter G, et al. Panning phage antibody libraries on cells: isolation of human fab fragments against ovarian carcinoma using guided selection. Cancer Res. 1998:58:991-6.

50. Ab O, Whiteman KR, Bartle LM, Sun X, Singh R, Tavares D, et al. IMGN853, a folate receptor-a (FRa)-targeting antibody-drug conjugate, exhibits potent targeted antitumor activity against FRa-expressing tumors. Mol Cancer Ther. 2015;14:1605-13.

51. Reddy JA, Dorton R, Bloomfield A, Nelson M, Dircksen C, Vetzel M, et al. Preclinical evaluation of EC1456, a folate-tubulysin anti-cancer therapeutic. Sci Rep. 2018;8:8943,018-27320-5.

52. Naumann RW, Coleman RL, Burger RA, Sausville EA, Kutarska E, Ghamande SA, et al. PRECEDENT: a randomized phase II trial comparing Vintafolide (EC145) and Pegylated liposomal doxorubicin (PLD) in combination versus PLD alone in patients with platinum-resistant ovarian Cancer. J Clin Oncol. 2013;31:4400-6

53. Taflin H, Odin E, Derwinger K, Carlsson G, Gustavsson B, Wettergren Y. Relationship between folate concentration and expression of folateassociated genes in tissue and plasma after intraoperative administration of leucovorin in patients with colorectal cancer. Cancer Chemother Pharmacol. 2018:82:987-97.

54. Giovannetti E, Zucali PA, Assaraf YG, Funel N, Gemelli M, Stark M, et al. Role of proton-coupled folate transporter in pemetrexed resistance of mesothelioma: clinical evidence and new pharmacological tools. Ann Oncol. 2017;28:2725-32.

55. Song DG, Ye Q, Poussin M, Harms GM, Figini M, Powell DJ Jr. CD27 costimulation augments the survival and antitumor activity of redirected human T cells in vivo. Blood. 2012;119:696-706.

56. Lynn RC, Feng Y, Schutsky K, Poussin M, Kalota A, Dimitrov DS, et al. Highaffinity FRß-specific CAR T cells eradicate AML and normal myeloid lineage without HSC toxicity. Leukemia. 2016;30:1355-64.

57. Zhao R, Matherly LH, Goldman ID. Membrane transporters and folate homeostasis: intestinal absorption and transport into systemic compartments and tissues. Expert Rev Mol Med. 2009;11:e4.

58. Moore KN, Martin LP, O'Malley DM, Matulonis UA, Konner JA, Vergote I, et al. A review of mirvetuximab soravtansine in the treatment of platinumresistant ovarian cancer. Future Oncol. 2018;14:123-36.

59. Chalouni C, Doll S. Fate of antibody-drug conjugates in Cancer cells. J Exp Clin Cancer Res. 2018;37:20.

60. Townsend MH, Shrestha G, Robison RA, O'Neill KL. The expansion of targetable biomarkers for CAR T cell therapy. J Exp Clin Cancer Res. 2018;37:163

61. Schutsky K, Song DG, Lynn R, Smith JB, Poussin M, Figini M, et al. Rigorous optimization and validation of potent RNA CAR T cell therapy for the treatment of common epithelial cancers expressing folate receptor. Oncotarget. 2015;6:28911-28

62. Lynn RC, Poussin M, Kalota A, Feng Y, Low PS, Dimitrov DS, et al. Targeting of folate receptor $\beta$ on acute myeloid leukemia blasts with chimeric antigen receptor-expressing T cells. Blood. 2015;125:3466-77.

63. Yao S, Li L, Su XT, Wang K, Lu ZJ, Yuan CZ, et al. Development and evaluation of novel tumor-targeting paclitaxel-loaded nano-carriers for ovarian cancer treatment: in vitro and in vivo. J Exp Clin Cancer Res. 2018;37:29.

Ready to submit your research? Choose BMC and benefit from:

- fast, convenient online submission

- thorough peer review by experienced researchers in your field

- rapid publication on acceptance

- support for research data, including large and complex data types

- gold Open Access which fosters wider collaboration and increased citations

- maximum visibility for your research: over $100 \mathrm{M}$ website views per year

At BMC, research is always in progress.

Learn more biomedcentral.com/submission 